\title{
On the KdV Equation with Hysteresis
}

\author{
Marius-Florinel Ionescu ${ }^{1}$, Ligia Munteanu ${ }^{2}$, Veturia Chiroiu ${ }^{2 *}$ \\ ${ }^{1}$ Liceul Teoretic Ion Neculce, Bucharest, Romania \\ ${ }^{2}$ Institute of Solid Mechanics of the Romanian Academy, Bucharest, Romania \\ E-mail: veturiachiroiu@yahoo.com
}

Received December 29, 2010; revised January 16, 2011; accepted January 20, 2011

\begin{abstract}
This paper discusses the generalized play hysteresis operator in connection with the KdV equation. Results from the nonlinear semigroup theory are applied to assure the existence and uniqueness. The KdV equation with hysteresis is reduced to a system of differential inclusions and solved.
\end{abstract}

Keywords: Hysteresis Operator, KdV Equations with Hysteresis

\section{Introduction}

The word hysteresis originates in the Greek word hysterein, which is translated as to be behind or to come later. The related Greek word hysteresis means shortcoming or lag in arrival. Ewing in 1885 [1] defined hysteresis as follows: When there are two quantities $M$ and $N$ such that cyclic variations of $N$ cause cyclic variations of $M$, then if the changes of $M$ lag behind those of $N$, we may say that there is hysteresis in the relation of $M$ and $N$. This definition gives an idea of what hysteresis is. The hysteresis is coupling to PDEs with hysteresis, which arise in many fields such plasticity, dynamics with friction, ferromagnetism, ferroelectricity, superconductivity, adsorption and desorption, biology, chemistry and economics.

We note that the phenomenon is similar to the standard approach within continuum mechanics related to the sixth Hilbert problem [2]. Hilbert's sixth problem is to axiomatize those branches of science in which mathematics is prevalent. It occurs on the list of Hilbert's problems given out in 1900. The explicit statement is the Mathematical Treatment of the Axioms of Physics. The investigations on the foundations of geometry suggest the problem: To treat in the same manner, by means of axioms, those physical sciences in which already today mathematics plays an important part; in the first rank are the theory of probabilities and mechanics.

In the 1970s, Krasnoselski1 and Pokrovski1 studied the concept of hysteresis operator, acting in spaces of time dependent functions [2]. Further researches were developed in a series of monographies dedicated to the hysteresis in connection with PDEs and applicative prob- lems [3-5]. A useful survey can be found in [6]. Nonlinear semigroup theory in a Hilbert space was developed by Kōmura [7] and extended to Banach spaces by Crandal and Liggett [8] and Barbu [9]. Nonlinear semigroup theory represents a widely used tool for solving nonlinear PDEs. A survey of basic relevant results from a nonlinear semigroup theory, formulated generally in a Banach space is presented in $[10,11]$.

Several models of mechanical and magnetic hysteresis may be represented via analogical models, namely the rheological models in mechanics, circuital models in electromagnetism, by arranging elementary components in series and/or in parallel [12-14]. These models consist of a family of elements, which can be interpreted as representing the mesoscopic structure of a composite material. Therefore, the procedure known as homogenization may be applied to provide an averaged representation of the system [15].

In this paper, the generalized play operator is analyzed in connection with the $\mathrm{KdV}$ equation. The problem is reduced to a system of differential inclusions and solved. This work is in the framework of the Visintin researches on models of hysteresis phenomena and on related PDEs [5,6,16-19].

\section{Hysteresis Operators}

In order to simplify the meaning of the hysteresis, let us consider a system whose the state is characterized by two scalar variables, the input function $u(t)$ and the output function $w(t)$, confined to a set $L \subset R^{2} . \forall t \in[0, T]$. The function $w(t)$ depends on the previous evolution of $u(t)$ (memory effect) and on the initial state $w_{0}$, 
such as

$$
\begin{aligned}
& w(t)=A\left(u, w_{0}\right)(t), \forall t \in[0, T], \\
& \left(u(0), w_{0}\right) \in L, A\left(u, w_{0}\right)(0)=w_{0},
\end{aligned}
$$

where $A\left(u, w_{0}\right)$ is a memory operator defined in a Banach space of time-dependent functions for any fixed $w_{0}$. The memory operator is causal: for $\forall\left(u_{1}, w_{0}\right)$, $\left(u_{2}, w_{0}\right)$ with $u_{1}=u_{2}$ in $[0, T]$, then $A\left(u_{1}, w_{0}\right)(t)=$ $A\left(u_{2}, w_{0}\right)(t)$. Most typical hysteresis phenomena exhibit not purely rate-independent memory and as consequence, the rate-dependent effects are superposed to hysteresis. In the memory rate-dependent case, the hysteresis operator is not invariant with reference to any increasing diffeomorphism $\varphi:[0, T] \rightarrow[0, T]$, i.e. $A\left(u \circ \varphi, w_{0}\right) \neq A\left(u, w_{0}\right) \circ \varphi, \forall t \in[0, T]$.

In the following we present the generalized play operator $w:=A\left(u, w_{0}\right): R^{+} \rightarrow R$ defined in the sense of Visintin (Figure 1). Let $u(t)$ be any continuous, piecewise linear function on $R^{+}$, linear on $\left[t_{i-1}, t_{i}\right]$, $i=1,2, \cdots$ We define $w(t)=A\left(u, w_{0}\right)(t)$ by

$$
\begin{aligned}
w(t)= & \min \left\{\gamma_{l}(u(0)), \max \left\{\gamma_{r}(u(0)), w_{0}\right\}\right\} \\
& \text { for } t=0 \text { and } w_{0} \in R, \\
w(t)= & \min \left\{\gamma_{l}\left(u\left(t_{i}\right)\right), \max \left\{\gamma_{r}\left(u\left(t_{i}\right)\right), w\left(t_{i-1}\right)\right\}\right\} \\
& \text { for } t \in\left(t_{i-1}, t_{i}\right), i=1,2, \cdots,
\end{aligned}
$$

where $\gamma_{l}, \gamma_{r}: R \rightarrow R$ are maximal monotone, possible multivalued functions with

$$
\inf \gamma_{r}(u) \leq \sup \gamma_{l}(u), \forall u \in R
$$

Note that $w(0)=w_{0}$ only if $\gamma_{r}(u(0)) \leq w_{0} \leq \gamma_{l}(u(0))$. The classical play operator can be obtained from the general play operator by choosing

$$
\gamma_{l}(u)=u+r, \gamma_{r}(u)=u-r,
$$

with $r \geq 0$ a parameter, $u(t)$ a continuous input function on $[0, T]$ and $w_{r 0} \in[-r, r]$ an initial state. Figure 2 presents the play operator with threshold $r$.

The hysteresis relationship with the PDEs can be written as [10].

$$
w(x, t)=\left[A\left(u(x, \cdots), w_{0}(x)\right)\right](t) \text { in } Q=\Omega \times[0, T],
$$

where $\Omega$ is a bounded subset of $R^{n}$. The generalized play operator discussed in this paper is dissipative, in the sense that $\|(\lambda I-A) x\| \geq \lambda\|x\|$ for $\forall \lambda>0$, where $I$ is the identity mapping.

The PDEs with hysteresis can be transformed into systems of differential inclusions. The generalized play operator can be defined as a solution in the Sobolev space $W^{1,1}(0, T), w \in W^{1,1}(0, T)$ of a variational inclusion of the type.

$$
w_{, t} \in \phi(u, w) \text { in }(0, T), w(0)=w_{0} .
$$

The norm in $W^{1,1}(0, T)$ is defined as

$$
\|f\|_{k, p}=\left(\sum_{i=0}^{k}\left\|f^{(i)}\right\|_{p}^{p}\right)^{1 / p}=\left(\left.\sum_{i=0}^{k} \int f^{(i)}\right|^{p} \mathrm{~d} t\right)^{1 / p} .
$$

The rate- independent differential inclusion is

$$
\begin{aligned}
& w_{, t} \in \phi(u, w) \\
& = \begin{cases}\{\infty\} & \text { if } w<\inf \gamma_{r}(u), \\
{[0,+\infty]} & \text { if } w \in \gamma_{r}(u) / \gamma_{l}(u), \\
\{0\} & \text { if } \sup \gamma_{r}(u)<w<\inf \gamma_{l}(u), \\
{[-\infty, 0]} & \text { if } w \in \gamma_{l}(u) / \gamma_{r}(u), \\
\{-\infty\} & \text { if } w>\sup \gamma_{r}(u), \\
{[-\infty,+\infty]} & \text { if } w \in \gamma_{l}(u) \cap \gamma_{r}(u) .\end{cases}
\end{aligned}
$$

If $\gamma_{r}$ and $\gamma_{l}$ are Lipschitz-continuous, then the generalized play operator transforms $(u, v) \in W^{1,1}(0, T 0 \times \mathrm{R}$ into the unique function $w \in W^{1,1}(0, T)$ such that $w(0)$ is the projection of $v$ into $\left[\gamma_{r}\left(u(0), \gamma_{l}(u(0)]\right.\right.$ and (7) is satisfied. The operator can be extended to $C^{0}([0, T]) \times \mathrm{R}$, and it is equivalent to a variational inequality [20].

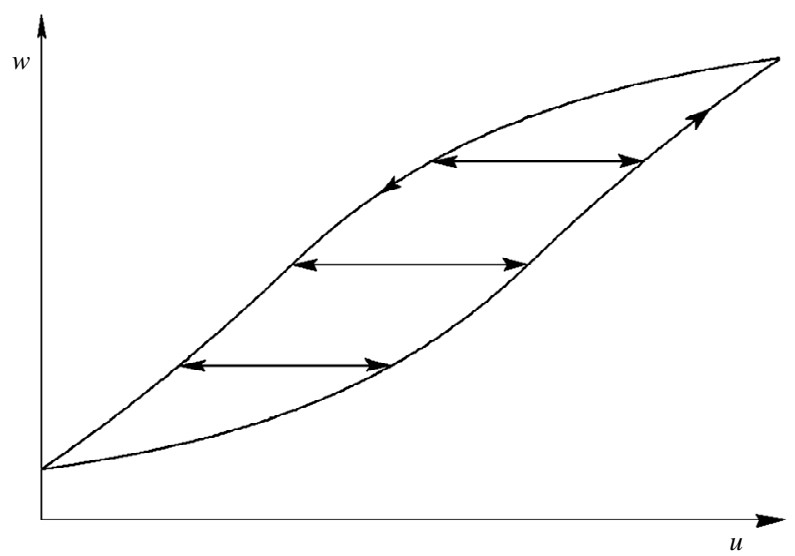

Figure 1. The generalized play operator.

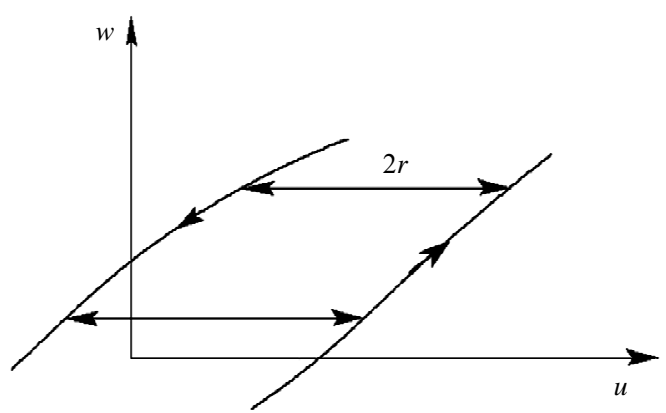

Figure 2. The play operator with threshold $r$. 

[10]

We present here one example of PDE with hysteresis

$$
(u+w)_{, t}-\Delta u=f \text { in } Q,
$$

related to a generalized play operator (3) by (6), is formally equivalent to $[5,10]$

$$
u_{, t}+\xi-\Delta u=f, w_{, t}-\xi=0, \xi \in \phi(u, w) \text { in } Q,
$$

where $\phi$ is defined by (7) and comma represents the differentiation with respect to the specified variable. The Cauchy problem for (9) coupled with homogeneous Dirichlet boundary conditions as

$$
F \in U_{, t}+A_{1} U \text { in } Q, U(0)=U_{0} \text { in } \Omega,
$$

where

$$
\begin{aligned}
& U=(u, w)^{T}, F=(f, 0)^{T}, \\
& A_{1} U=(\xi-\Delta u,-\xi)^{T}, \xi \in \phi(U) \cap R .
\end{aligned}
$$

At the end of this section some results of the nonlinear semigroup theory are presented in the spirit of [10]. Let $B$ be a Banach space, $A$ nonlinear and multivalued hysteresis operator $A: D(A) \subset B \rightarrow B$ is accretive if

$$
\left\|u_{1}-u_{2}\right\|_{B} \leq\left\|u_{1}-u_{2}+\lambda\left(v_{1}-v_{2}\right)\right\|_{B}, \forall \lambda>0 .
$$

Definition 2. Let $B$ be a Banach space, the hysteresis operator $A$ is called $m$-accretive if $\operatorname{Rg}(I+\lambda A)=B$, $\forall \lambda>0$

Suppose that the derivative in the evolution equation can be approximated by a backward-difference quotient of step size $h>0$ and $f$ by a step functions $f_{k}^{h}$. We have

$$
\begin{aligned}
& f_{k}^{h} \in \frac{u_{k}^{h}-u_{k-1}^{h}}{h}+A\left(u_{k}^{h}\right), k=1,2, \cdots, u_{0}^{h}=u_{0}, \\
& u_{k}^{h}(t)=u_{k}^{h}, \text { for } k h \leq t<(k+1) h .
\end{aligned}
$$

The scheme (13) is uniquely solved recursively and the Crandall-Liggett theorem holds:

Theorem 1. (Crandall-Liggett) [8]: If $A$ is $m$-accretive, $f \in L^{1}(0, T, B)$ and $u_{0} \in \bar{D}(A)$ and $f^{h} \rightarrow f$ in $L^{1}(0, T, B)$, then $u^{h} \rightarrow u$ uniformly as $h \rightarrow 0$ and $u \in C(0, T, B)$.

Theorem 2: If $A$ is $m$-accretive, $f \in L^{1}(0, T, B)$ and $u_{0} \in \bar{D}(A)$, then the Cauchy problem

$$
f \in u_{, t}+A(u(t)), u(0)=u_{0} .
$$

has one and only one integral solution $u$. For $f=0$, we have $u=S(t) u_{0}$, where $S(t)$ is a nonlinear semigroup of contractions generated by the operator $A$. If $f$ has bounded variation in $[0, T]$ and $u_{0} \in D(A)$, then the integral solution is Lipschitz continuous.

Definition 3. The function $u$ is an integral solution of (15) in the sense of Benilan if 1) $u:[0, T] \rightarrow B$ is continuous; 2) $u(t) \in \bar{D}(A)$ for any $t \in[0, T]$; and 3 ) $u(0)=u_{0}$ and

$$
\begin{aligned}
& \left\|u\left(t_{2}-v\right)\right\|_{B}^{2} \leq\left\|u\left(t_{1}-v\right)\right\|_{B}^{2} \\
& +2 \int_{t_{1}}^{t_{2}} \lim _{\lambda \rightarrow 0} \frac{\| u(\tau-v)+\lambda(f(\tau)-z))\left\|_{B}^{2}-\right\| u(\tau-v) \|_{B}^{2}}{2 \lambda} \mathrm{d} \tau .
\end{aligned}
$$

\section{The KdV Equation with Hysteresis}

Amplitude equations governing the non-linear resonant interaction of equatorial baroclinic and barotropic Rossby waves were derived by Majda and Biello [21,22] and used as a model for long range interactions between the tropical and mid-latitude troposphere. Exploiting the fact that some of the Rossby waves can resonantly interact, Majda and Biello [23] developed a small amplitude theory of nearly dispersionless, long equatorial Rossby waves. The analytic solitary wave solutions can be constructed with the functional form of the KdV soliton. These results inspire us to analyse the $\mathrm{KdV}$ equation from the point of view of the hysteresis of waves.

The KdV equation with hysteresis can be written under the form

$$
\begin{aligned}
& q_{, t}(x, t)=q_{, x x x}(x, t)-6 q(x, t) q_{, x}(x, t) \\
& \text { in } Q=[-\infty, \infty] \times[0, T], \\
& q(x, t)=u(x, t)+w(x, t), \\
& \lim _{x \rightarrow \pm \infty} u(x, t)=0, \\
& w(x, t)=A\left(u(x, t), w_{0}\right), w_{, t} \in \phi(u, w), \\
& w(x, 0)=w_{0}(x) \text { in }(0, T),
\end{aligned}
$$

where $\phi(u, w)$ is defined by (7). The hysteresis relation (5) representing a generalized play is also valid.

For $w=0$, the exact solution of (17) is obtained by choosing the solution under the form $u(x, t)=z(x-\beta t)$. The exact solution is a solitary wave

$$
u(x, t)=\frac{\beta}{2} \operatorname{sech}^{2}\left[\frac{\sqrt{\beta}}{2}(x-\beta t)\right]
$$

[24]. In order to have a real solution the quantity $\beta$ must be a positive number. For $\beta>0$ the solitary wave moves to the right, and the amplitude of the solitary wave is proportional to the speed which is indicated by the value of $\beta$. Thus larger amplitude solitary waves move with a higher speed than smaller amplitude waves.

To solve (17) we use the Lax formalism [25]. Equation (17) can be described by two operators depending of the hysteresis operator $A=A\left(u(x, t), w_{0}\right)$

$$
L(A)=-\partial_{x x}+q, M(A)=-4 \partial_{x x x}+3\left(q \partial_{x}+\partial_{x} q\right) .
$$


The operator $L(A)$ characterizes the spectral problem

$$
L(A) \psi(x, t, \lambda)=\lambda \psi(x, t, \lambda),
$$

and the operator $M(A)$ characterizes the $t$-evolution of the wavefunction $\psi(x, t, \lambda)$

$$
\psi_{, t}(x, t, \lambda)=-M(A) \psi(x, t, \lambda) .
$$

The compatibility of (19) and (20) when $\lambda$ is not dependent of $t$ implies the Lax equation $L_{, t}=[L, M]$.

The algebraic properties which derive from the existence of the operator $L(A)$ refer to the existence of a recursor operator $\Lambda(A)$, and the existence of Bäcklung and Darboux transformations $[24,25]$.

Starting from (19), we can look for operators $M_{j}(A)$ such that to have satisfied

$$
L_{, t}=\left[L, M_{j}\right] .
$$

Consider the following set of operator equations

$$
[L, M]=V(A),[L, \tilde{M}]=\tilde{V}(A),
$$

where $V$ and $\tilde{V}$ are scalar functions for the operators $M$ and $\tilde{M}$. Taking account the structure of the operator $L(A)$, we have

$$
\tilde{M}(A)=L(A) M(A)+F(A) \partial x+G(A),
$$

where $F$ and $G$ are scalar functions of $A$ and its derivative and of $V$ defined by (22).

From Equations (22) and (23) it results that $\tilde{V}$ can be expressed as a recursor operator $\Lambda(A)$ on the function $V$ and depending on the hysteresis operator $A$

$$
\Lambda V(x, t)=-\frac{1}{4} V_{, x x}(x, t)+q(x, t) V(x, t)-\frac{1}{2} q_{, x}(x, t) \int_{x}^{\infty} V(y, t) \mathrm{d} y .
$$

By using (24), the problem (17) becomes

$$
\begin{aligned}
& (u+w)_{, t}=\Lambda(A)(u+w)_{, x} \text { in } Q=[-\infty, \infty] \times[0, T], \\
& \lim _{x \rightarrow \pm \infty} u(x, t)=0, \\
& w=A\left(u, w_{0}\right), w_{, t} \in \phi(u, w), \\
& w(x, 0)=w_{0}(x)=\sin (k x) \text { in }(0, T),
\end{aligned}
$$

where $\phi(u, w)$ is defined by (7). In the spirit of Visintin [5], the problem (25) is formally equivalent to a system of differential inclusions

$$
\begin{aligned}
& u_{, t}+\xi=\Lambda(A)(u+A)_{, x} \text { in } Q=[-\infty, \infty] \times[0, T], \\
& \lim _{x \rightarrow \pm \infty} u(x, t)=0, \\
& w_{, t}-\xi=0, \xi \in \phi(u, w), \\
& w(x, 0)=w_{0}(x)=\sin (k x) \text { in } Q,
\end{aligned}
$$

where $\phi(u, w)$ is defined by (7).

Figure 3 illustrates the hysteretic solution of the pro- blem (26) for $k=3, t \in[0,7], x \in[-40,40]$. For $t<6$ the curve is a helix, then the solution exhibits several hysteretic loops for $t \geq 6$. The transition from a helix into the hysteresis loops is greatly aided by the excitation history expressed as a superposition of solitary waves. The transition instantaneously occurs as in the case of the climatologically appropriate mean winds and shears.

For $t \geq 20$, an intriguing aspect of the interaction appears by splitting of the hysteresis loop into two distinctive branches. Figure 4 presents these two branches for $t \in[20,100], \quad x \in[-30,30]$. The solution varies between two hysteresis branches depending of the excitation history. Such branches, the splitting and formation of a double-sides comblike hysteresis loops have been observed experimentally [26].

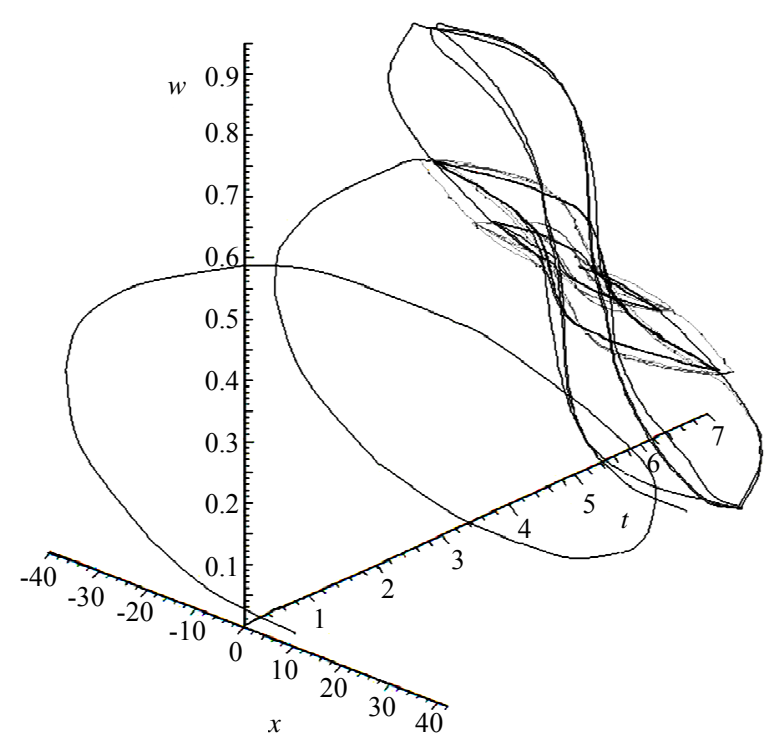

Figure 3. The hysteretic solution $w(x, t)$ of the problem (26) $t \in[0,7]$.

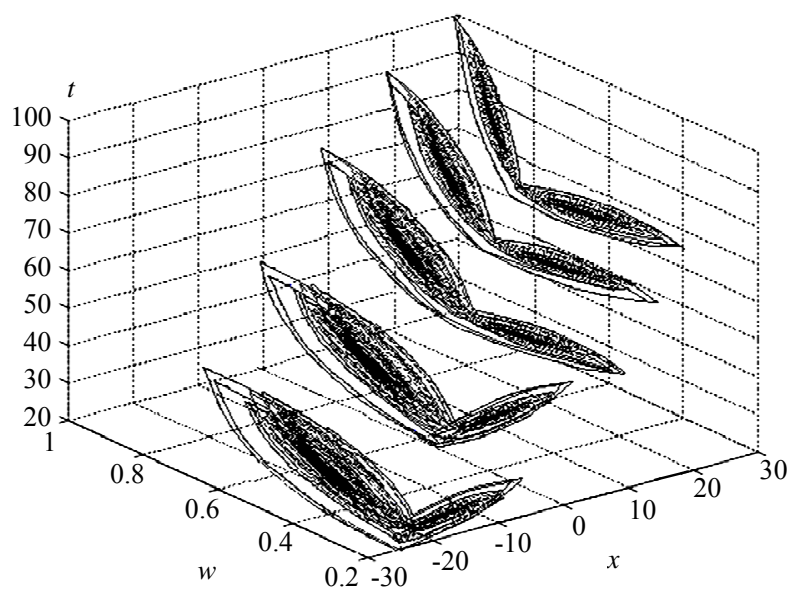

Figure 4. The hysteretic solution $w(x, t)$ of the problem (26) $t \in[20,100]$. 


\section{Conclusions}

This paper is aimed to outline some of the basic elements of the hysteresis operators in connection with PDEs. The construction of the $\mathrm{KdV}$ equation with hysteresis is just an example of a more general method developed by Visintin $[5,6]$. The KdV equation with hysteresis is reduced to a system of differential inclusions and solved.

\section{Acknowledgement}

The authors are grateful to the National Authority for Scientific Research (ANCS, UEFISCSU), Romania, through PN-II research project nr. 745/2009, code ID_1391/2008.

\section{References}

[1] J. A. Ewing, "Experimental Research in Magnetism," Philosophical Transactions of the Royal Society of London, Vol. 176, No. 2, 1895, pp. 131-159.

[2] M. A. Kranoselskii and A. V. Pokrovskii, "Systems with Hysteresis," Springer, Berlin, 1989.

[3] M. Brokate and J. Sprekels, "Hysteresis and Phase Transitions," Springer, Berlin, 1996.

[4] P. Krećí, "Convexity, Hysteresis and Dissipation in Hyperbolic Equations," Gakkotosho, Tokyo, 1997.

[5] A. Visintin, "Differential Models of Hysteresis," Springer-Verlag, Berlin 1995.

[6] A. Visintin, "Quasi-Linear Hyperbolic Equations with Hysteresis," Annales de l' Institute Henri Poincaré, Nonlinear Analysis, Vol. 19, No. 4, 2002, pp. 451-476. doi:10.1016/S0294-1449(01)00086-5

[7] Y. Kōmura, "Nonlinear Semi-Groups in Hilbert Space," Journal of the Mathematical Society of Japan, Vol. 19, No. 4, 1967, pp. 493-507. doi:10.2969/jmsj/01940493

[8] M. C. Crandall and T. M. Liggett, "Generation of Semigroups of Nonlinear Transformations on General Banach spaces," American Journal of Mathematics, Vol. 93, No. 2, April 1971, pp. 265-298. doi:10.2307/2373376

[9] V. Barbu, "Nonlinear Semigroups and Differential Equations in Banach Spaces," Noordhoff, Leyden, 1976.

[10] J. Kopfová, "Nonlinear Semigroup Methods in Problems with Hysteresis," Discrete and Continuous Dynamical Systems Supplement, 2007, pp. 580-589.

[11] A. Visintin, "Hysteresis and Semigroups," In: A. Visintin, Ed., Models of Hysteresis, Longman, Harlow, 1993, pp. 192-206.

[12] D. Damjanovic, "Hysteresis in Piezoelectric and Ferroelectric Materials," In: G. Bertotti, I. Mayergoyz, Eds., The Science of Hysteresis, Elsevier, 2006, pp. 338-46.

[13] I. D. Mayergoyz, "Mathematical Models of Hysteresis and Their Applications," Elsevier, Amsterdam, 2003.
[14] G. Bertotti, "Hysteresis in Magnetism," Academic Press, Boston, 1998.

[15] A. Visintin, "Homogenization of Some Models of Hysteresis," Physica B: Condensed Matter, Vol. 403, No. 2-3, February 2008, pp. 245-249. doi:10.1016/j.physb.2007.08. 020

[16] V. Mosnegutu and V. Chiroiu, "On the Dynamics of Systems with Friction," Proceedings of the Romanian Academy, Series A: Mathematics, Physics, Technical Sciences, Information Science, Vol. 11, No. 1, 2010, pp. 63-68.

[17] P. P.Teodorescu, V. Chiroiu, L. Munteanu and D. Dumitriu, "On the Resonance Wave Interaction Phenomenon," Revue Roumaine des Sciences Techniques, série de Mécanique Appliquée, Vol. 55, No. 1, 2010, pp. 31-38.

[18] V. Preda, M. F. Ionescu, V. Chiroiu and T. Sireteanu, "A Preisach Model for the Analysis of the Hysteretic Phenomena," Revue Roumaine des Sciences Techniques, série de Mécanique Appliquée, Vol. 55, No. 3, 2010, pp. $75-86$.

[19] A. S. Gliozzi, L. Munteanu, T. Sireteanu and V. Chiroiu, "An Identification Problem from Input-Output Data," Revue Roumaine des Sciences Techniques, série de Mécanique Appliquée, Vol. 55, No. 3, 2010, pp. 54-67.

[20] A. Visintin, "Mathematical models of hysteresis," In: G. Bertotti, I. Mayergoyz, Eds., The Science of Hysteresis, Elsevier, 2006, pp. 1-123.

[21] J. A. Biello and A. J. Majda, "Boundary Layer Dissipation and the Nonlinear Interaction of Equatorial Baroclinic and Barotropic Rossby Waves," Geophysical \& Astrophysical Fluid Dynamics, Vol. 98, No. 2, 2004, pp. 85-127. doi:10.1080/03091920410001686712

[22] J. A. Biello and A. J. Majda, "The Effect of Meridional and Vertical Shear on the Interaction of Equatorial Baroclinic and Barotropic Rossby Waves," Studies in Applied Mathematics, Vol. 112, No. 4, 2004, pp. 341-390. doi:10. 1111/j.0022-2526.2004.01518.x

[23] A. J. Majda and J. A. Biello, "The Nonlinear Interaction of Barotropic and Equatorial Baroclinic Rossby Waves," Journal of the Atmospheric Sciences, Vol. 60, No.15, 2003, pp. 1809-1821. doi:10.1175/1520-0469(2003)060< 1809:TNIOBA>2.0.CO;2

[24] L. Munteanu and S. Donescu, "Introduction to Soliton Theory: Applications to Mechanics," Book Series Fundamental Theories of Physics, Vol. 143, Kluwer Academic Publishers, Dordrecht, 2004.

[25] D. Levi and O. Ragnisco, "Dressing Method and Bäcklund and Darboux Transfomations," CRM Proceedings and Lecture Notes, Centre de Recherches Mathématiques, Vol. 29, 2001, pp. 29-51.

[26] R. V. Lapshin, "Analytical Model for the Approximation of Hysteresis Loop and Its Application to the Scanning Tunneling Microscope," Review of Scientific Instruments, Vol. 66, No. 9, 1995, pp. 4718-4730. doi:10.1063/1.1145 314 